\title{
THE IMPACT OF HOT JUPITERS ON THE SPIN-DOWN OF THEIR HOST STARS
}

\author{
O. Cohen ${ }^{1}$, J. J. Drake ${ }^{1}$, V. L. Kashyap ${ }^{1}$, I. V. SoKolov ${ }^{2}$, and T. I. Gombosi ${ }^{2}$ \\ ${ }^{1}$ Harvard-Smithsonian Center for Astrophysics, 60 Garden St. Cambridge, MA 02138, USA \\ ${ }^{2}$ Center for Space Environment Modeling, University of Michigan, 2455 Hayward St., Ann Arbor, MI 48109, USA \\ Received 2010 September 5; accepted 2010 September 29; published 2010 October 12
}

\begin{abstract}
We present a numerical magnetohydrodynamic study of the dependence of stellar mass and angular momentum loss rates on the orbital distance to close-in giant planets. We find that the mass loss rate drops by a factor of $\approx 1.5-2$, while the angular momentum loss rate drops by a factor of $\approx 4$ as the distance decreases past the Alfvén surface. This reduction in angular momentum loss is due to the interaction between the stellar and planetary Alfvén surfaces, which modifies the global structure of the stellar corona and stellar wind on the hemisphere facing the planet, as well as on the opposite hemisphere. The simulation also shows that the magnitude of change in angular momentum loss rate depends mostly on the strength of the planetary magnetic field and not on its polarity. The interaction, however, begins at greater separation if the overall field topology of the star and the planet are anti-aligned. Our results are consistent with evidence for excess angular momentum in stars harboring close-in giant planets and show that the reduction in wind-driven angular momentum loss can compete with, and perhaps dominate, spin-up due to tidal interaction.
\end{abstract}

Key words: planet-star interactions - stars: coronae - stars: magnetic field

Online-only material: color figures

\section{INTRODUCTION}

Nearly two decades after the first discovery of a planet orbiting a main-sequence star outside of the solar system (Mayor \& Queloz 1995), hundreds of other exoplanets have been observed (Mayor et al. 2003). Many of these planets have masses in the Jupiter class and orbit their host star at distances of $\lesssim 10$ stellar radii. Such massive close-in planets are also called "hot Jupiters."

Planets that are far out in the stellar system cannot significantly affect their host star via tidal (gravitational) interaction. They also cannot affect the star via magnetic interaction (even if the planet has a strong internal magnetic field) since the superAlfvénic stellar wind prevents the propagation of information back from the planet to the star along the field lines; the star is thus essentially blind to the existence of the planetary magnetic field. The conceptual boundary that separates the sub- and super-Alfvénic regimes is called the "Alfvén surface," located at a radial distance from the star known as the "Alfvén radius." This surface is defined as the collection of critical points at which the wind flow speed, $u$, is equal to the Alfvén speed, $u_{A}$, or in other words, the Alfvénic Mach number, $M_{A}=u / u_{A}=1$. In the case of close-in planetary orbits at distances comparable to or less than the stellar Alfvén radius, magnetic interactions are more easily facilitated and can, at least in principle, induce a feedback on the star via star-planet magnetic interaction.

Signatures of star-planet interaction have been observed through local modulations in the Ca II $\mathrm{K}$ emission line-an indicator of chromospheric activity-in several planetary systems (Shkolnik et al. 2005a, 2005b, 2008; see also Hartman 2010). In addition, an apparent enhancement in stellar activity in stars with close-in planets compared with stars with planets located at greater orbital radii appears in the form of an overall increase in observed X-ray (Kashyap et al. 2008; see, however, Poppenhaeger et al. 2010) and UV (Shkolnik 2010) flux.

Recently, Pont (2009) performed an empirical study between stellar rotation rate, orbital semimajor axis, stellar and planetary radii, and the planet-to-star mass ratio. This study suggests that stars that harbor hot Jupiters rotate faster than stars with planets located at larger orbital separation or stars without planets at all. Pont (2009) followed (Mardling \& Lin 2002) in suggesting that the excess in stellar angular momentum is due to tidal torques that synchronize the stellar rotation with the planetary orbital motion. It is also possible to view this result as a reduction in the angular momentum lost from stars with hot Jupiters, rather than as the host stars being spun up. Lanza (2010) suggests that in many observed planetary systems the synchronization timescale is too long for tidal interaction to cause this excess in stellar angular momentum. Instead, he proposes that the angular momentum excess is due to star-planet magnetic interaction that leads to a reduction of magnetic breaking and angular momentum loss to the stellar wind (Weber \& Davis 1967). He concludes that both mechanisms can take part in the process of angular momentum transfer and that the dominant of the two mechanisms depends on the particular set of stellar-planetary parameters, as well as the stellar type.

In this Letter, we present magnetohydrodynamic (MHD) numerical simulations of the star-planet interaction, where we focus on calculating the stellar angular momentum loss rate for a representative set of stellar and planetary parameters as a function of the planetary orbital radius. We describe the simulation details in Section 2 and the results in Section 3. We summarize in Section 4.

\section{SIMULATION}

The simulations described here were performed in the same manner as in Cohen et al. (2009b). The model used solves the set of MHD equations, based on the surface distribution of the stellar magnetic field, and provides a self-consistent, steadystate solution for the stellar corona and stellar wind (Cohen et al. 2007). The planet is represented by a dipole magnetic field with boundary conditions typical of Jupiter. Since the simulation provides the solution for the density, velocity, magnetic field, and pressure, we can calculate the realistic Alfvén surface and 

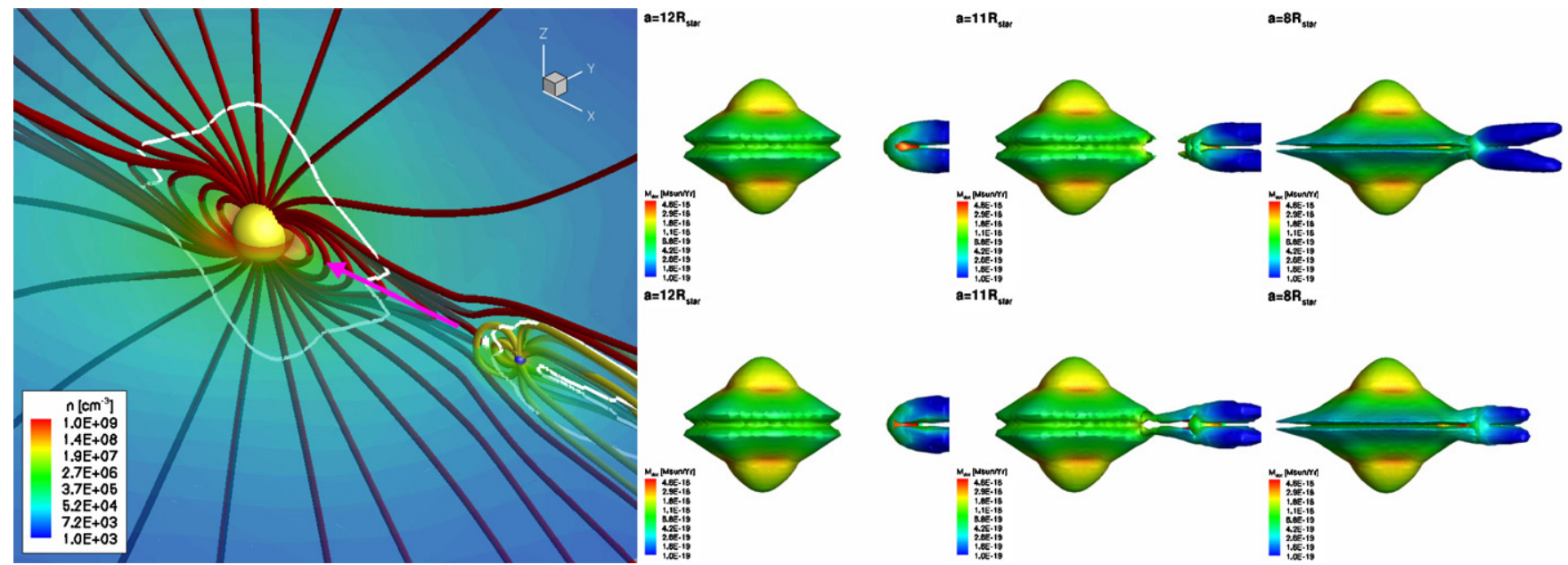

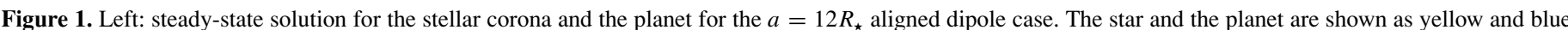

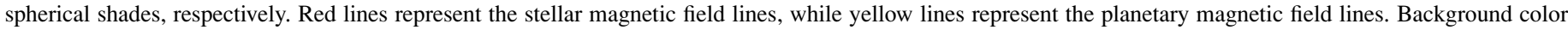

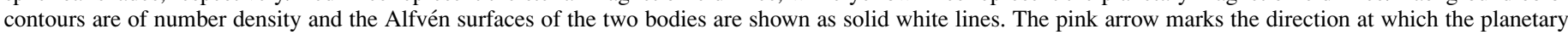

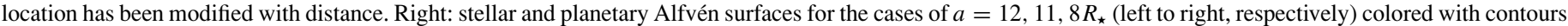

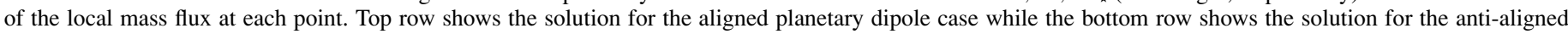
planetary dipole case.

(A color version of this figure is available in the online journal.)

hence the stellar angular momentum loss rate (see Cohen et al. 2009a for a detailed description of this procedure).

We adopt some of the stellar properties of the well-observed HD 189733 system $\left(R_{\star}=0.76 R_{\odot}, M_{\star}=0.82 M_{\odot}\right.$ (Mayor et al. 2003), and $P_{\text {rot }}=11.95$ days (Fares et al. 2010)). Fares et al. (2010) have recently investigated the surface magnetic field distribution of HD 189733; here we adopt an idealized dipolar stellar magnetic field in order not to complicate any modulation of model results with planetary distance. The planetary field is assumed to be dipolar as well, with equatorial field of $B_{p}=1 \mathrm{G}$. The choice of a field somewhat lower than that of Jupiter is based on the expected slower rotation of close-in planets as a result of tidal locking (Sánchez-Lavega 2004), and the observed inverse correlation between planetary magnetic field strength and rotation period (e.g., Durand-Manterola 2009).

In addition to the stellar Alfvén surface, we expect the planet to possess an analogous surface due to planetary outflow. In this simulation, we mimic the planetary outflow by introducing the planet as a mass source for the plasma outflow, where the flow rate depends on the particular choice of the planetary boundary conditions. The planetary radius used here is $R_{p}=1.95 R_{J}\left(R_{J}\right.$ is Jupiter's radius) with boundary conditions for the density, $n_{p}=10^{7} \mathrm{~s} \mathrm{~cm}^{-3}$, and temperature, $T_{p}=10^{4} \mathrm{~K}$. This particular choice of parameters produces a planetary outflow of about $50 \mathrm{~km} \mathrm{~s}^{-1}$.

Here, we study modulations in stellar angular momentum loss rate as a function of the semimajor axis, $a$. Once the stellar Alfvén surface is defined, we calculate the mass and angular momentum loss rates which depend on the topology of this surface. We explore values of $a$ ranging from $12 R_{\star}$ to $3 R_{\star}$, and calculate the loss rates for each case. We omit the case of $a=5 R_{\star}$, since at this distance the planet is located exactly at the stellar Alfvén surface. This causes the stellar Alfvén surface to be an open surface with the grid space of the simulations and the integration required for the loss rate calculations is illconditioned. We also test how the orientation of the planetary magnetic field affects the loss rates by defining a planetary dipole magnetic field that is aligned or anti-aligned to the stellar dipolar magnetic field.
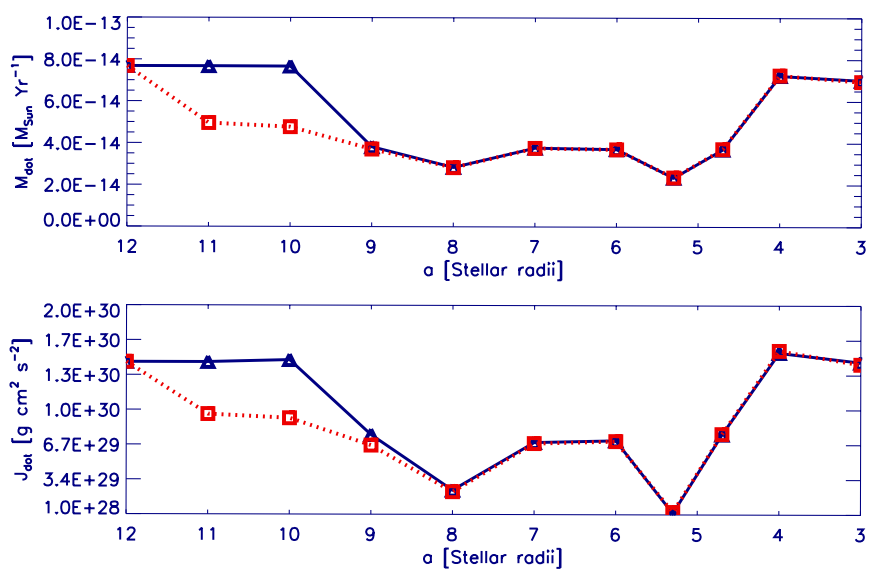

Figure 2. Mass loss rates, $M_{\mathrm{dot}}$ (top, units of solar mass per year), and angular momentum loss rates, $J_{\text {dot }}$ (bottom, units of $\left.\left(g_{\mathrm{cm}^{2} \mathrm{~s}}^{-2}\right)\right)$, for the case of aligned planetary dipole (solid blue line, triangles) and anti-aligned planetary dipole (dashed red line, squares).

(A color version of this figure is available in the online journal.)

\section{RESULTS AND DISCUSSION}

The MHD solution for the particular case of an aligned planetary dipole and semimajor axis $a=12 R_{\star}$ is illustrated in the left panel of Figure 1. Also shown with an arrow is the range of semimajor axis investigated. The six panels on the right of Figure 1 show the stellar and planetary Alfvén surfaces for different values of $a$ and for aligned and anti-aligned dipoles.

The results for the mass and angular momentum loss rates as a function of $a$ are shown in Figure 2. It can be seen that the mass loss rate drops by about a factor of $1.5-2$ at $a=9 R_{\star}$ for the aligned case and at $a=11 R_{\star}$ for the anti-aligned case. The trends in the plot for the angular momentum loss rate are similar, but here the change reaches a factor of 4 at $a=8 R_{\star}$. The reason for this change is clearly seen in Figure 3, where we show the mass flux distribution, as well as the location of the Alfvén surface over the star-planet meridional plain for the cases of $a=12 R_{\star}$ and $a=6 R_{\star}$. In the case of $a=12 R_{\star}$, the 


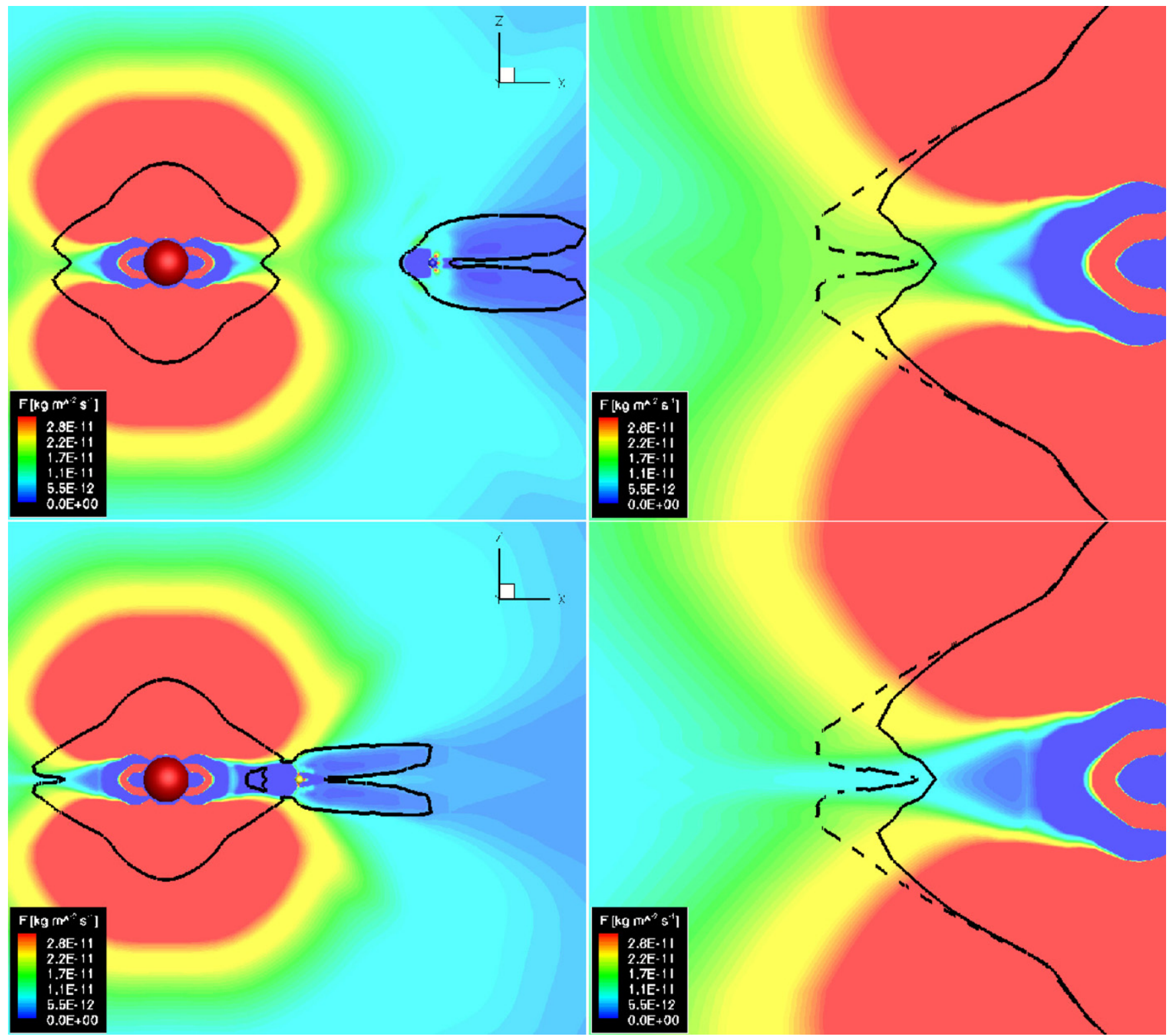

Figure 3. Mass flux distribution (color contours) and the location of the Alfvén surface (black line) displayed on the $y=0$ (star-planet) meridional plain. The solution for the $a=12 R_{\star}$ case is shown on the top panels while the solution for the $a=6 R_{\star}$ case is shown on the bottom panels. Left panels show the global solution with the star as a red sphere, and the planet as a blue sphere $\left(a=12 R_{\star}\right)$ or yellow sphere $\left(a=6 R_{\star}\right)$. The right panels show a zoom on the vicinity of the stellar corona hemisphere which is not occupied by the planet. In both right panels, the Alfvén surface for the $a=12 R_{\star}$ case is shown as a solid line, while the Alfvén surface for the $a=6 R_{\star}$ case is shown as a dashed line.

(A color version of this figure is available in the online journal.)

front of the planetary Alfvén surface is beyond the stellar one, so that the existence of the planet does not affect the structure of the stellar corona. The large-scale structure of the corona is disrupted by the planet in the case of $a=6 R_{\star}$, due to the fact that the planet is located inside the stellar Alfvén surface so the stellar corona and stellar wind "feel" the obstacle (i.e., the planet and its magnetosphere). In particular, both on the planetary side and on the opposite side, the mass flux near the equatorial region is significantly reduced due to the existence of the planet. This explains the $50 \%$ reduction in mass flux when the planet affects the corona. The fact that the coronal side opposite to the planet is modified demonstrates that the effect of the planet on the corona is global. We would like to point out that another reference simulation with a different set of stellar and planetary parameters resulted in the same trend, so this effect does not depend on the particular choice of parameter set. In addition, an initial result from a simulation of the HD 189733 planetary system, which includes realistic stellar magnetic field, as well as a planetary orbital motion resulted in a total mass flux of $6 \times 10^{-14} M_{\odot} \mathrm{yr}^{-1}$, which is close to the value of $4 \times 10^{-14} M_{\odot} \mathrm{yr}^{-1}$ obtained from this simulation for $a=9 R_{\star}$ (as observed for HD189733).

Since in our case the star and the planet are tidally locked, the planet blocks a significant amount of the stellar wind and prevents the stellar wind from opening up field lines and escaping (Cohen et al. 2009b). For the particular set of parameters used in our simulations, the planetary magnetosphere has a large enough cross section to block the stellar wind over a significant area. A planet without a significant internal magnetic field would not only be too small to affect the mass flux through the Alfvén 
surface, but would probably suffer from a strong erosion by the stellar wind. In our simulations, the radius of the cross section of the planetary magnetosphere is not larger than about 1-2 stellar radii. Therefore, the area that is blocked by the planet is not more than about $25 \%-30 \%$ of the total area of the stellar Alfvén surface. Nevertheless, the whole coronal hemisphere that faces the planet is affected, so that the stellar mass loss rate decreases by approximately a factor of 1.5-2 as a result of the interaction with the planetary magnetosphere.

The four right panels of Figure 2 show that the planet begins to affect the corona when the planetary Alfvén surface (and not the planet itself) starts to interact with the stellar one. They also shows that the two surfaces merge at greater separation for the case of an anti-aligned planetary dipole as compared with the aligned case. This explains the smaller value of $a$ at which a drop in mass and angular momentum loss rates compared to the aligned dipole case is seen. This merging of the surfaces at smaller $a$ is due to particular magnetic field lines that connect the planetary magnetic field and the stellar one.

The amount of change in the loss rates depends mostly on the size of the planetary magnetosphere, which is determined by the particular choice of planetary parameters. The alignment of the planetary field has only a minor effect on the change in angular momentum loss. This is due to the fact that the wind is blocked primarily by the magnetic pressure of the planetary field, which depends on the magnitude of the field but not on its polarity. It is most likely that a tilted planetary dipole will produce only slightly different result, depending on the tilt angle.

Pont (2009) presented empirical evidence for "excess" rotation in stars hosting close-in planets. This was interpreted in terms of tidal forces acting toward spin-orbit synchronization and transfer of orbital to rotational angular momentum. While such angular momentum exchange doubtless takes place, the MHD models presented here demonstrate that the stellar angular momentum loss rate through magnetized winds also likely plays a significant role. Close-in planets reduce this angular momentum loss and reduce stellar spin-down. The effect is large enough - a factor of up to 4-that it could dominate the observed rotation excesses. As noted by Pont (2009), spin-up by tidal effects requires substantial orbital decay since the dissipation of the protoplanetary disk. The reduced magnetic braking effect found here would imply that much less severe orbital decay would be required.

Full understanding of the distribution of planetary orbits and stellar rotation rates likely requires consideration of both tidal and magnetic angular momentum loss and exchange. Improvements in the MHD modeling presented can be made at the expense of substantial computational cost by including time-dependent effects of the star-planet interaction using more realistic surface magnetic field distributions.

\section{CONCLUSIONS}

In this Letter, we calculate variations in stellar angular momentum loss rate of a star hosting a hot Jupiter planet as a function of the planetary orbital distance. We show that once the stellar and planetary Alfvén surfaces interact with each other, the stellar wind topology in the hemisphere facing the planet changes and the angular momentum loss to the wind decreases. The simulation also shows that this interaction begins at a greater distance if the overall field topology of the star and the planet are opposite, though the magnitude of change in angular momentum loss rate depends mostly on the size of the planetary magnetosphere. This reduction in angular momentum loss for close-in planets likely contributes to, and perhaps even dominates, the observed excess rotation found in stars with close-in planets.

We thank an unknown referee for his/hers useful comments. O.C. is supported by SHINE through NSF ATM-0823592 grant and by NASA-LWSTRT Grant NNG05GM44G. J.J.D. and V.L.K. were funded by the NASA contract NAS8-39073 to the Chandra X-ray Center. Simulation results were obtained using the Space Weather Modeling Framework, developed by the Center for Space Environment Modeling, at the University of Michigan with funding support from NASA ESS, NASA ESTO-CT, NSF KDI, and DoD MURI.

\section{REFERENCES}

Cohen, O., Drake, J. J., Kashyap, V. L., \& Gombosi, T. I. 2009a, ApJ, 699, 1501

Cohen, O., Drake, J. J., Kashyap, V. L., Saar, S. H., Sokolov, I. V., Manchester, W. B., Hansen, K. C., \& Gombosi, T. I. 2009b, ApJ, 704, L85

Cohen, O., et al. 2007, ApJ, 654, L163

Durand-Manterola, H. J. 2009, Planet. Space Sci., 57, 1405

Fares, R., et al. 2010, MNRAS, 406, 409

Hartman, J. D. 2010, ApJ, 717, L138

Kashyap, V. L., Drake, J. J., \& Saar, S. H. 2008, ApJ, 687, 1339

Lanza, A. F. 2010, A\&A, 512, A77

Mardling, R. A., \& Lin, D. N. C. 2002, ApJ, 573, 829

Mayor, M., Naef, D., Pepe, F., Queloz, D., Santos, N., \& Udry, S. 2003, The Geneva Extrasolar Planet Search Programmes, http://exoplanets.eu

Mayor, M., \& Queloz, D. 1995, Nature, 378, 355

Pont, F. 2009, MNRAS, 396, 1789

Poppenhaeger, K., Robrade, J., \& Schmitt, J. H. M. M. 2010, A\&A, 515, A98

Sánchez-Lavega, A. 2004, ApJ, 609, L87

Shkolnik, E. 2010, BAAS, 41, 531

Shkolnik, E., Bohlender, D. A., Walker, G. A. H., \& Collier Cameron, A. 2008, ApJ, 676, 628

Shkolnik, E., Walker, G. A. H., Bohlender, D. A., Gu, P.-G., \& Kurster, M. 2005a, ApJ, 622, 1075

Shkolnik, E., Walker, G. A. H., Rucinski, S. M., Bohlender, D. A., \& Davidge, T. J. 2005b, AJ, 130, 799

Weber, E. J., \& Davis, L. J. 1967, ApJ, 148, 217 